rev.relac.int.estrateg.segur.8(1):113-133,2013

\title{
EL MILA.
}

\section{MERCADO DE INTEGRACIÓN ENTRE CHILE, PERÚ Y COLOMBIA*}

\author{
William Vargas Pulido** \\ José Bayardo Martínez ${ }^{* * *}$
}

\section{RESUMEN}

El crecimiento económico está unido al fenómeno de la globalización y conlleva un efecto positivo de la integración de los mercados. La integración de las bolsas de valores de Perú, Chile y Colombia generó unos efectos dentro y fuera del mercado, que impactan a los participantes ocasionando unos beneficios y unas dificultades con consecuencias en la operatividad de este mercado.

Palabras clave: Integración Financiera, Mercado Integrado Latinoamericano, Negocios Regionales.

* Este articulo es producto del proyecto Integración, relaciones y negocios internacionales, Chile-Perú-Colombia, código EES 928, inscrito en la Vicerrectoría de Investigaciones de la universidad Militar Nueva Granada.

** Economista. Máster en Finanzas y Dirección Financiera (Universidad del País Vasco). Docente investigador de la Maestría en Relaciones y Negocios internacionales de la Universidad Militar. william.vargas@unimilitar.edu.co

*** Administrador de Empresas. Máster en Administración de Empresas (Universidad Externado). Docente de la Universidad Sergio Arboleda. josebayardoster@gmail.com 


\title{
THE MILA: MARKET INTEGRATION BETWEEN CHILE, PERU AND COLOMBIA
}

\begin{abstract}
Economic growth is linked to the phenomenon of globalization and carries a positive effect on market unification. The integration of the stock markets of Peru, Chile and Colombia carried consequences both inside and outside of the markets themselves, which have an impact on the participants, generating benefits and difficulties, with consequences in the operational aspects of this market.
\end{abstract}

Keywords: Financial Integration, Integrated Latin American Market, Regional Businesses.

\section{O MILA MERCADO DE INTEGRAÇÃO ENTRE O CHILE, O PERU E A COLÔMBIA}

\section{RESUMO}

O crescimento econômico está ligado ao fenômeno da globalização e determina um efeito positivo da integração dos mercados. A integração das bolsas de valores do Peru, Chile e Colômbia gerou efeitos dentro e fora do mercado, que impactam os participantes e ocasionam alguns benefícios e dificuldades com consequências para a operatividade deste mercado.

Palavras-chave: Integração Financeira, Mercado Integrado da América Latina, Negociações Regionais.

\section{INTRODUCCIÓN}

El aspecto económico que inspiró la globalización es, sin lugar a dudas, el crecimiento empresarial (Mateus y Brasset, 2002). Este objetivo es perseguido por la mayoría de las empresas y ha sido un tema central en el campo de la planeación estratégica. La integración, a su vez, puede ser una buena estrategia para el crecimiento en las ventas en la medida en que se desarrollan nuevos mercados; pero este crecimiento depende de tratados entre naciones, así como de las políticas internas de los países frente a las multinacionales e inversionistas extranjeros.

Las empresas, en su plan estratégico, plantean que deben tener una ventaja competitiva sostenible a largo plazo (Gun y Resnick, 2006). Para lograr esas ventajas tratarán de encontrar nuevos mercados que generen crecimientos permanentes en las ventas, porque la ausencia de crecimiento podría ser uno de los mayores riesgos que pueden correr. La expansión de una empresa 
también conlleva importantes peligros y riesgos como, por ejemplo, la inversión en sectores en los que el éxito competitivo depende de la posesión de recursos, capacidades y conocimientos de los que la empresa no dispone; otro problema puede ser el logro de una dimensión empresarial superior a lo que la empresa no sea capaz de gestionar conduciéndolos a la pérdida de valor.

Para evitar esta situación las empresas utilizan estrategias como la integración horizontal y vertical, con las cuales pueden ingresar a un mercado nuevo con menor riesgo y a mayor velocidad (Hill y Jones, 2009).

En la última década este tipo de integraciones se ha incrementado en las empresas de los mercados financieros, sobre todo en las bolsas de valores. De esta manera se han venido conformando grupos económicos empresariales de gran poder operativo, financiero y tecnológico. Tales desarrollos permiten a los inversionistas tener acceso a los mercados de otros países, diversificando los productos y generando un mayor volumen de empresas. En consecuencia, la integración de los mercados de valores se ha convertido en un proceso ineludible en un mundo globalizado, donde la circulación de capitales se sitúa a la vanguardia de la mundialización. América Latina dio este paso con las bolsas de Chile, Perú y Colombia. Desde mayo de 2011, tres empresas privadas se integraron en un solo mercado, denominado Mercado Integrado Latinoamericano (MILA), acuerdo que permite en su primera fase las operaciones financieras de compra y venta de acciones de las compañías listadas en los tres mercados.

La integración de estas tres bolsas busca generar varios efectos cruciales en el crecimiento económico. Entre ellos, el que los mercados y los intermediarios financieros eficaces movilicen el ahorro y lo dirijan hacia la inversión, de tal manera que favorezcan así la productividad y la innovación; también ocurre que el funcionamiento de los mercados de capitales logra desarrollar economías de escala (a través de la financiación con tasas de interés más bajas, permitiendo a las empresas incrementar la producción); por otra parte, desde la demanda (los inversionistas), se observa una externalidad de red, ya que las ganancias obtenidas por un inversor por el hecho de participar en un mercado aumentan con el número de participantes. Así, la consolidación de los mercados aumenta la liquidez de un título e incita a los operadores a realizar más transacciones, amplificando el efecto inicial de consolidación. Otro efecto consiste en que la reunión de las transacciones y de las operaciones de pago-entrega en plataformas integradas reduce los costos de transacción a beneficio de los inversores, así como de las empresas de mercado y de los emisores. Estos diferentes efectos son reforzados por el desarrollo de las tecnologías de la comunicación y de la información (Kern, 2008).

Este artículo busca evidenciar los efectos estructurales que ocasiona la integración, y lo hace a partir de los hechos ocurridos entre los entes reguladores de los mercados de valores, los depósitos centrales de valores y las bolsas de valores de Chile, Perú y Colombia entre mediados de 2009 (época en que se iniciaron conversaciones de integración) y 2012 (cuando ya está en funcionamiento). 
Para poder desarrollar este objetivo, se requiere, en primer lugar, una revisión de los fundamentos teóricos de la integración económica. En segundo lugar, es necesario confrontar la teoría con los efectos ocasionados por el MILA, teniendo en cuenta cuáles han generado un beneficio y cuáles están teniendo dificultades en la operatividad de este mercado.

\section{FUNDAMENTOS TEÓRICOS}

Primero veamos algunos conceptos de integración. La palabra integración proviene del latín integratio, que quiere decir renovación. Según el profesor Fritz Machlup, el término integratio, usado en el sentido de combinar las partes en un todo, data del año 1600 (Conesa, 1982). Una de las definiciones más conocida es la Bela Balassa, quien considera la integración como un proceso o estado de cosas por las cuales diferentes naciones deciden formar un grupo regional. Divide, además, la integración en distintos niveles, como son la integración comercial, la integración de factores, la integración a nivel de políticas y la integración total (Conesa, et al., 1982).

Basado en el análisis de la integración, Enderlein recomienda a los agentes políticos y los reguladores adoptar un enfoque holístico cuando evalúen la integración: "La integración puede ser una oportunidad para el diseño de políticas para crear un mercado financiero más estable e integrado" (Enderlein, 2011).

Hay que tener en cuenta que la integración económica es un medio para alcanzar el desarrollo económico, porque permite satisfacer en mayor medida las necesidades de consumo de los habitantes de un país. En consecuencia, contribuye a un mayor bienestar general (Corbo, 1993). Esto se hace a través de diferentes medios para lograrlo. Por un lado, a través de la mayor división del trabajo que un mercado ampliado permite; por otro, mediante la posibilidad de utilizar las economías de escala en un mercado con un número mayor de consumidores.

De lo anterior podemos decir que la división del trabajo y las economías de escala se relacionan estrechamente, porque cuando una empresa toma la decisión de realizar economía de escala, busca reducir sus costos de producción para hacerse más competitiva en un mercado; por consiguiente, la empresa necesitará personal más calificado, y esto significa más división del trabajo. Por tal razón, estas dos actividades van muy de la mano en una integración económica (Agacino, 1997).

Teniendo en cuenta estos dos conceptos, veamos cado uno de ellos con mayor amplitud. Para el caso de las economías de escala (también llamadas de rendimientos decrecientes), esta se logra con el desarrollo o implementación de tecnología. Consiste en aplicar los métodos y la tecnología moderna que ayudan a la producción en masa, es decir, la producción en grandes cantidades, situación que le permite a la empresa bajar considerablemente los costos (Cline, 1982). Esto quiere decir que, se fundamenta de forma que la producción es mas eficiente cuanto 
mayor es la escala en la que se lleva a cabo. Cuando hay economías de escala, la duplicación de los factores de producción de una industria provoca que la producción aumente más del doble (Krugman \& Obstfeld, 2008).

Consecuentemente, las economías de escala tienen un impacto sobre la estructura del mercado y dependen del incremento de la producción que es necesario para reducir el costo medio ${ }^{1}$. Por ello debemos considerar dos tipos de economías de escala: una hace referencia a las economías de escala externa, aquella que se produce cuando el costo unitario ${ }^{2}$ depende del tamaño de la industria, pero no necesariamente del tamaño de sus empresas. La otra se refiere a las economías de escala internas que se producen cuando el costo unitario depende del tamaño de una empresa individual, pero no necesariamente de la industria (Krugman, et al., 2008).

Por otra parte, en cuanto a la división del trabajo, el economista Adam Smith en su libro La riqueza de las naciones, establece que la división del trabajo está limitada por la extensión del mercado (Smith, 1958). La división del trabajo permite aumentar la productividad de la economía. Por consiguiente, para que la mayor eficiencia que produce esta división del trabajo pueda verificarse en la práctica, es necesario que haya un mercado grande. De ese modo, el principio de la división del trabajo de Smith ha sido aplicado por las diferentes economías, las economías capitalistas. Por ejemplo, en Estados Unidos, en la constitución de 1787, se prohíben los derechos de importación (aranceles) entre los estados de la Unión. Este es un concepto fundamental, que le permitió a Estados Unidos establecer un mercado ampliado y comenzar a desarrollarse hasta constituirse en la primera potencia económica del mundo (Beard, 1953).

Para el caso de los países socialistas, la división del trabajo se conoce como un factor que aumenta la eficiencia económica (Robson, 1980). Por ejemplo, en este tipo de economías, los países se asociaron en una especie de mercado común, llamado Consejo de Asistencia Económica Mutua, que pretendía establecer un gran mercado con la Unión Soviética, Polonia, Alemania Oriental, Bulgaria, Rumania y Checoslovaquia (Conesa, et al., 1982). En el mundo socialista se reconoce ampliamente que la división del trabajo aumenta considerablemente la eficiencia económica. En conclusión, el concepto de división del trabajo es universal y es utilizado por todas las ideologías políticas; es un concepto vital para el análisis de la integración económica.

Un tercer concepto es la teoría de la ventaja comparativa expuesta por el economista David Ricardo en su libro Principios de economía política y tributación en el año de 1817. Dice Ricardo que cada país debe especializarse en aquellos productos que podemos producir mejor, adquirir una gran destreza en esa producción, exportar, vender ese producto; y en los productos que no sea fuerte la producción, importarlos (Ricardo, 1959). De esta forma se podrá elevar el

1. Se define como el costo total de producción dividido por el número de unidades producidas.

2. El costo unitario se calcula dividiendo el costo de producción total por las unidades equivalentes de producción. 
nivel de vida. Este principio es para que las naciones lo apliquen, pero se aplica también a cada individuo, quien debe desarrollar unas capacidades que le permitan ser competitivo frente a los otros individuos. Por lo tanto, debe especializase en una actividad en la cual tenga ciertas ventajas.

Existe otro concepto, el de la igualación de los precios de los factores por la vía del comercio; es decir, que la intensificación del comercio entre dos países conduce a que la remuneración de los factores de la producción entre ambos tienda a igualarse (Samuelson, 1949). Pero este concepto está sujeto a que los factores de la producción, trabajo y capital tengan facilidad de movilidad, es decir, a la posibilidad de transferencia de factores entre los distintos países.

Un quinto concepto, muy importante para la integración económica, es la competencia, que consiste en que en un mercado se encuentren empresas en diferentes condiciones, que pueden ser monopolios, oligopolios y competencia monopolística. En este último tipo de mercado hay mayor competencia entre las empresas, los precios son más estables, y el número de empresas en la industria es elevado. Para el caso de los monopolios y oligopolios es diferente, ya que la competencia es menor, las empresas son pocas. Las empresas pueden influir sobre los precios de los productos; en estos tres tipos de competencia las empresas están permanentemente innovando, mejorando sus métodos de producción y siempre busca disminuir los costos. Pero lo fundamental de la competencia consiste en que requiere de un mercado ampliado y no una o dos empresas en un mercado nacional estrecho y pequeño (Corden, Werner \& Max, 1974). En consecuencia, la competencia obliga a las empresas a reducir los costos, a hacer que el empresario piense en la adopción de nuevos métodos de producción y a que esté siempre atento a la posibilidad de integrar nuevas tecnologías a la producción. Todo con el fin de hacerse más competitivo, permitiéndole así un incremento en las utilidades, incremento que se reflejará finalmente en el crecimiento económico del país. En síntesis, la competencia está dada por varios aspectos que, fundamentalmente, la determinan el número de empresas en un mercado. Igualmente, esa integración ayuda al crecimiento económico por la vía de mayor eficiencia que provoca la competencia.

Por otra parte, existen varias formas de integración económica de los Estados, tales como: zonas de preferencias, zonas de libre comercio, uniones aduaneras, mercado común y comunidad económica. Para el caso de nuestro estudio, nos vamos a enfocar en el mercado común. Existe tal mercado común cuando, además de libre circulación de los bienes y tarifa externa común, existe libre circulación de los factores de producción, es decir, del trabajo y del capital (Conesa, et al., 1982).

En cambio, por el lado del sector privado, se realizan integraciones de diferentes maneras, porque las empresas no siempre pueden desarrollar internamente todos los recursos que necesitan para ser competitivas. Este problema se ve agravado por la inexistencia de un mercado 
para este tipo de recursos de carácter estratégico, que suelen ser idiosincrásicos (específicos a una empresa) y, por tanto, imperfectamente imitables e imperfectamente móviles (Barney, 1991; Peteraf, 1993). Por esta situación, ante la carencia de recursos de esta naturaleza, las empresas se ven abocadas a la realización de algún tipo de combinación empresarial para proveerse de ellos (Chi, 1994). Este recurso de las combinaciones empresariales será mayor en la medida que las empresas estén sometidas a cambios en el entorno. Lo que normalmente cambian son las bases de la competencia en un sector, al aumentar los recursos necesarios para competir (García, 1999). A las combinaciones empresariales se les denominan integraciones y son de dos tipos, de adquisiciones y de alianzas.

De acuerdo a lo anterior, se presentan diferencias entre estos dos tipos de integración. La primera, las adquisiciones, implican la integración de más recursos que las alianzas (Hennart \& Reddy, 1997). Hay que tener en cuenta que, por un lado, las adquisiciones obligan a las compañías a integrar personas, sistemas organizativos y culturas diferentes, los que, normalmente, son un desafío para las empresas involucradas (Haspeslangh \& Jemison, 1991). Por otro lado, mientras que las adquisiciones afectan a todos los recursos de las empresas que se unen, las alianzas solo afectan a ciertos recursos. Esto aparentemente nos indica que hacer alianzas es más fácil que hacer adquisiciones, y no es así. En investigaciones realizadas sobre las alianzas se encontraron que tienen problemas relacionados con el comportamiento de oportunistas y con los objetivos contradictorios de los socios (Ring \& Van de Ven, 1994; Doz, 1996; Madhok \& Tallman, 1998; Ariño \& de la Torre, 1998). La segunda tiene que ver con el hecho de que cuando hay una adquisición existe una única dirección que se va a encargar de la gestión de los recursos que se ponen en común, mientras que en las alianzas la dirección de la empresa será, generalmente, compartida por varias personas de una u otra forma (Menguzzato, 1995; Rialp \& Salas, 2002). La tercera se refiere a que mientras en una adquisición la empresa adquiriente debe pagar por el valor total de los activos de la empresa adquirida, en las alianzas no es necesaria tal contraprestación, porque se basan en el acceso reciproco a recursos por parte de los socios (Hennart, 1988: 370). Por ultimo, las adquisiciones son operaciones con mayor grado de irreversibilidad que las alianzas. En las alianzas se integran recursos, pero son aportes de cada una de las empresas. Por lo general se hacen aportes en especie más que en dinero.

\section{EL MERCADO INTEGRADO LATINOAMERICANO, MILA}

La consolidación de las bolsas se ha convertido en un proceso ineludible en un mundo globalizado, donde la circulación de capitales se sitúa a la vanguardia de la mundialización. Las finanzas, se convierte en una herramienta estratégica y con alto valor añadido, y cuya rimpacto social es elevada. Es en este contexto que hay que considerar el papel y la evolución de las bolsas (Hamon, Jacquillat y Saint, 2003). América Latina no puede quedar fuera de este movimiento, que conlleva este desafío de la integración. 
En los últimos diez años ha habido una tendencia creciente a la integración de los mercados bursátiles, y, en especial, en los mercados europeos y estadounidenses, integración que es llevada a cabo fuera de las fronteras de los países de origen. La internacionalización de las bolsas de valores ha tomado la forma de mercados ampliados con un mayor número de oferta de productos financieros, buscando así incrementar el comercio a través de los flujos de inversiones. Esta internacionalización se ha hecho a través de operaciones de integración, fundamentalmente por adquisiciones. Ejemplo de esto son las bolsas de París, Amsterdam y Bruselas, que se integraron en el año 2000, para conformar Euronext, luego en el 2002 incorporaron la Bolsa de Valores de Lisboa y Oporto. Posteriormente, incorporaron la Liffe (Mercado Internacional de Futuros y Opciones de Londres). En el 2007, la Bolsa de Londres adquirió la Bolsa Italiana, incluyendo CC\&G (Central de Contraparte) y Monte Titoli (Depositario Central); en el 2007, también se dio la fusión más importante entre los mercados americano y europeo con las bolsas de Nyse (Bolsa de New York) y Euronex, que actualmente es conocida como NYSE Euronext, todas con fines de optimización de costos y de ampliar el mercado de renta variable.

En América Latina, como resultado de las reformas económicas, se ha avanzado en esa dirección, tanto en restablecer la estabilidad macroeconómica, como en desarrollar economías dinámicas lideradas por la iniciativa privada. La expansión del comercio y la liberalización de los mercados financieros domésticos han llevado, en forma creciente, a una expansión de los flujos financieros entre países de la región y de fuera de esta (Corbo, et al., 1997). La región se encuentra actualmente en un proceso de globalización de apertura de mercados de bienes y servicios y movilidad de capitales.

En ese contexto nace el MILA. Esto ocurre por varias necesidades, como la de integrar y generar un mercado más eficiente, amplio y variado, y también para conceder la oportunidad a los inversionistas de acceder a los mercados de Perú, Chile y Colombia, y a los emisores les permite una fuente de financiación más amplia. La integración de la Bolsa de Valores de Lima S.A (BVL), la Bolsa de Valores de Colombia (BVC) y la Bolsa de Comercio de Santiago (BCS), se dio el 29 de octubre de 2010. Este hecho consiste en una integración en forma de alianza de tres empresas privadas, y se produce a través del proceso denominado enrutamiento intermediado. Esto implica que las bolsas, al igual que sus depósitos de valores ${ }^{3}$ en los tres países se interconectaran conservando la independencia de sus plataformas de negociación. Todo ello sin modificar las reglas de negociación ni las de compensación y liquidación de operaciones vigentes de cada mercado, de acuerdo a la Resolución 107-2010-EF/94.01.1 de Perú (Romero, 2012).

3. Entidad privada que se dedica a custodiar y administrar una cartera de valores de acciones o bonos, aquellos que fueron adquiridos en una bolsa de valores por los inversionistas. 
Al respecto, el acuerdo de implementación, trazó el norte:

Implementar la primera fase del modelo de mercado integrado, definiendo los modelos de negociación, compensación, liquidación, tecnológico, jurídico y comercial. Dejo establecido que se permitirá el acceso al mercado integrado desde el mercado local, a través de un modelo intermediado con valor agregado. Este acuerdo dejo sentada la realización del proyecto en dos fases. Fase I: Facilitar el acceso al mercado local por parte de los intermediarios extranjeros, a través de mecanismos desarrollados por las bolsas y los depósitos, bajo la responsabilidad de un intermediario local en la negociación, compensación y liquidación de las operaciones. Fase II: Facilitar el acceso directo al mercado local por parte de los intermediarios extranjeros bajo las reglas de negociación estándar y un modelo de compensación y liquidación transfronterizo (Romero, et al., 2012).

Paralelamente, incorporamos el concepto de bolsa de valores. En los tres países las bolsas son organizaciones privadas reguladas por el Estado, son bolsas que se dedican a prestar servicios de comercialización de productos financieros. Atienden los mandatos de sus clientes, introducen ordenes y realizan negociaciones de compra y venta de acciones de sociedades o compañías anónimas, bonos públicos y privados y, además, realizan operaciones de coberturas por medio de derivados y operaciones de compra y venta de divisas. La negociación de los valores en los mercados bursátiles se hace con base en unos precios conocidos y en tiempo real; y el mecanismo de transacción son las plataformas electrónicas. Los participantes en las operaciones de las bolsas son los demandantes de capital (empresas, organismos públicos o privados), los oferentes de capital (ahorradores o inversionistas) (Kern, 2008). La negociación de valores en los mercados bursátiles se efectúa a través de unos intermediarios conocidos con el nombre de corredores, sociedades de corretaje de valores, casas de bolsa, agentes o comisionistas ${ }^{4}$, de acuerdo a la denominación que reciban en las leyes de cada país. Todos ellos son quienes hacen su labor a cambio de una comisión.

Por ejemplo, las bolsas más importantes del mundo, como la bolsa de Tokio, la de Londres, o la de Frankfurt están organizadas como mercados de subasta; pero el subastador, en estos casos, es un computador en vez de un especialista (Brealy, Myers \& Allen, 2006). Para el caso de estudio, las tres bolsas del MILA funcionan como subasta de acciones. El subastador es un computador que hace un ruteo de órdenes y cada bolsa posee su propia plataforma. Por consiguiente, en la

4. Empresas que sirven de intermediarias entre la bolsa de valores y los inversionistas. Se encargan de hacer las operaciones de compra y venta de valores que se realizan en la rueda de la bolsa de valores. 
alianza que se dio en cada mercado prevalecieron las plataformas y se adaptaron para que hubiese un calce.

\section{EFECTOS QUE HAN GENERADO BENEFICIO POR PARTE DEL MILA}

Para el caso del MILA, aunque es una alianza, la integración efectiva se produce cuando los socios vencen sus reticencias a compartir los activos necesarios para la obtención de la sinergia deseada con la operación. Para ello es necesaria la existencia de confianza, la que además actúa como un buen mecanismo de gobierno y garantía de la relación (Dyer y Singh, 1998). Según esto, en la integración del MILA se están compartiendo las plataformas de operación de cada bolsa, lo que es, quizás, el activo más importante para cada empresa. En el mismo sentido, en caso de no existir relaciones previas entre las compañías, la clave para generar esta confianza y obtener la información adecuada para organizar la cooperación, es el desarrollo de lo que Madhok denomina inversiones específicas en la relación. Esto implica, a su vez, dedicar tiempo, energía y esfuerzo para entender los objetivos de los socios y facilitar la interacción conjunta.

En el MILA las primeras conversaciones entre la bolsa de valores de Colombia y Perú se dieron a finales de 2007. Desde ese instante, se dieron varios procesos, como el conocimiento mutuo, así como entender la operatividad de cada empresa y analizar la economía de cada país. Posteriormente, se unió la bolsa de valores de Santiago. Fue cuando se dio el primer paso y se firmó el acuerdo de intención entre las tres bolsas, en septiembre de 2009. Posteriormente, el 8 de junio de 2010, se firmó el acuerdo de implementación y el 30 de mayo de 2011 se iniciaron operaciones.

Además, se ha producido una integración de los mercados regionales, atraídos por los de mayor dimensión, todo ello debido a la presión competitiva que soportan ante la globalización. De la misma forma, las bolsas integradas, buscan concentrar una mayor oferta de empresas cotizadas por acciones. Este incremento de empresas ofrecidas en el mercado de valores impacta directamente en la creación de valor, impulsando a las empresas oferentes de títulos a un espiral de crecimiento (Martin \& Téllez, 2006). Se puede afirmar que este es el primer impacto que causó la integración. Cada mercado, independientemente, estaba conformado de la siguiente forma: Chile tenía 227 empresas, Perú 235 y Colombia 84 cotizadas por acciones. Las tres bolsas, sumadas en el MILA, equivalen a 546 empresas en total. Finalmente, se cumplió uno de los objetivos propuestos en la integración, que era dar profundidad ${ }^{5}$ al mercado. Ver gráfico 1.

5. Consiste en tener mayores opciones para los participantes; es decir, más cantidad de productos en el mercado y, a la vez, más participantes.

EL MILA. MERCADO DE INTEGRACIÓN ENTRE CHILE, PERÚ Y COLOMBIA 


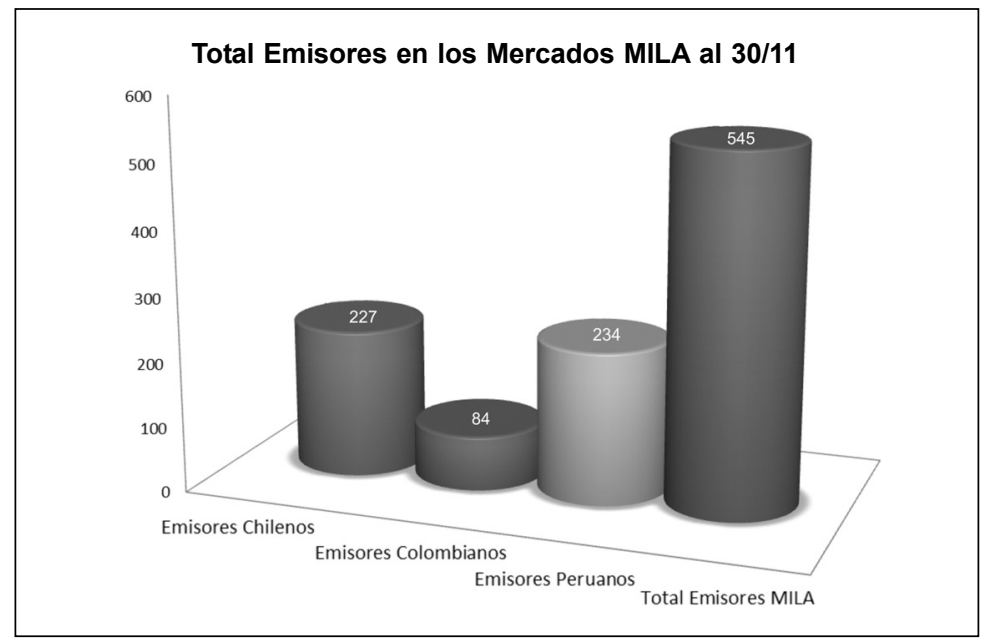

Gráfico 1. Total Emisores en los Mercados MILA al 30/11 Fuente: MILA News, diciembre de 2011.

Con la integración se generó una gran expectativa a nivel de los tres mercados, debido al incremento notable en el número de empresas en el mercado. Las empresas que rodean las bolsas de valores, como es el caso de las empresas comisionistas de bolsa, han realizado integraciones horizontales de alianzas con el fin de participar al interior de cada uno de los mercados. A diciembre de 2012 se han firmado 47 convenios (ver gráfico 2). Adicionalmente, se han realizado adquisiciones entre las diferentes comisionistas, dando el primer paso para generar economías de escala, como es el caso del banco peruano Credicorp, que compró a uno de los principales agentes de la Bolsa de Colombia, a Correval; también, el banco chileno Corbanca compró, en el 2011, al Banco Santander Colombia y, en el 2012, al Helm Bank.

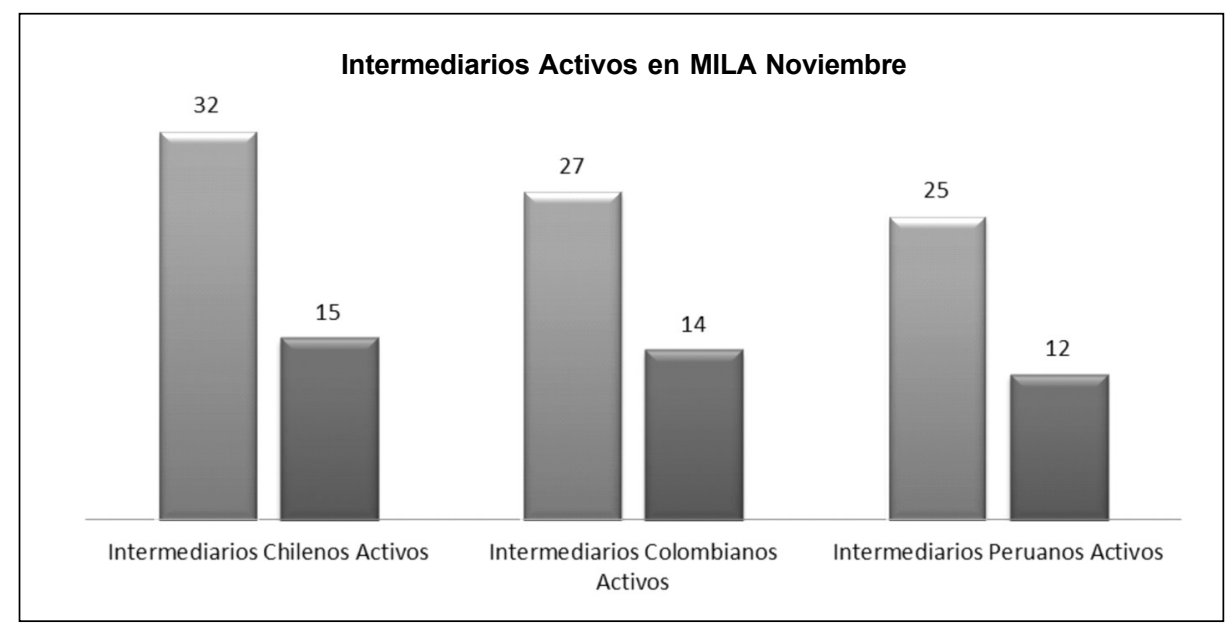

Gráfico 2. Intermediarios Activos en MILA Noviembre Fuente: Mila News, diciembre de 2012. 
Otro efecto que ocasionó la integración fue la complementación de los mercados, que consiste en agrupar a diferentes industrias y sectores económicos en un mismo lugar, lo que permite una mayor competencia. Las empresas no siempre pueden desarrollar internamente todos los recursos que necesitan para ser competitivas; tener nuevos mercados donde operar permite a las compañías incrementar sus posibilidades de crecimiento, pero, a la vez, les obliga a ser más competitivas.

El mercado también ha traído beneficios en relación con la división del trabajo, específicamente, porque los tres mercados se complementan en lo que tiene que ver con los diferentes tipos de empresas que hay en cada una de las bolsas (ver gráfico 3). Se requiere de personas calificadas y especializadas en los temas de cada sector empresarial como, por ejemplo, analistas financieros.

Otro aspecto fundamental que hay que tener en cuenta es la ventaja comparativa, en el que cada país debe especializarse en aquellos productos que puedan producir mejor; es decir, adquirir una gran destreza en la producción. Cada mercado tiene en cuenta sus recursos naturales (tierra), el dinero invertido (capital) en las diferentes empresas y el personal calificado (trabajo) en las diferentes áreas. Por lo tanto, cada empresa que participa en el mercado integrado tiene la posibilidad de complementar a los otros dos mercados y es, quizás, la oportunidad más importante que tienen los emisores para participar en estos nuevos mercados y lograr estrategias de crecimiento.

Otro caso más: la integración permite a los inversionistas diversificar ${ }^{6}$ sus portafolios ${ }^{7}$. El MILA ha logrado, fundamentalmente, que los inversionistas puedan diversificarse, porque cada país tiene unas fortalezas y debilidades en sus mercados internos. Por ejemplo, Chile está bastante desarrollado en la minería, sector retail y comercial; por el lado de Perú, está desarrollado en áreas de pesca y minería; y Colombia en los sectores del petróleo, financiero y eléctrico. Veamos en el Gráfico 3 cómo está conformada la estructura del mercado.

Los sectores más fortalecidos con la integración son el financiero, el de materiales y el comercio. Adicionalmente, hay sectores que no tenían ninguna participación en los mercados internos de cada país; pero, ahora con esta integración, permiten una complementación. Por ejemplo, Colombia no contaba con empresas de los sectores de la minería, telecomunicaciones y construcción.

A parte de eso, una de las situaciones más complejas de resolver para la integración era la liquidación de fondos y valores, una vez realizadas las operaciones. Debido a la existencia de

6. Consiste en invertir en varios productos financieros a la vez para no asumir el riesgo de un solo producto.

7. Muestra los productos financieros en los cuales se ha realizado una inversión de capital; también se le denomina cartera.

EL MILA. MERCADO DE INTEGRACIÓN ENTRE CHILE, PERÚ Y COLOMBIA 


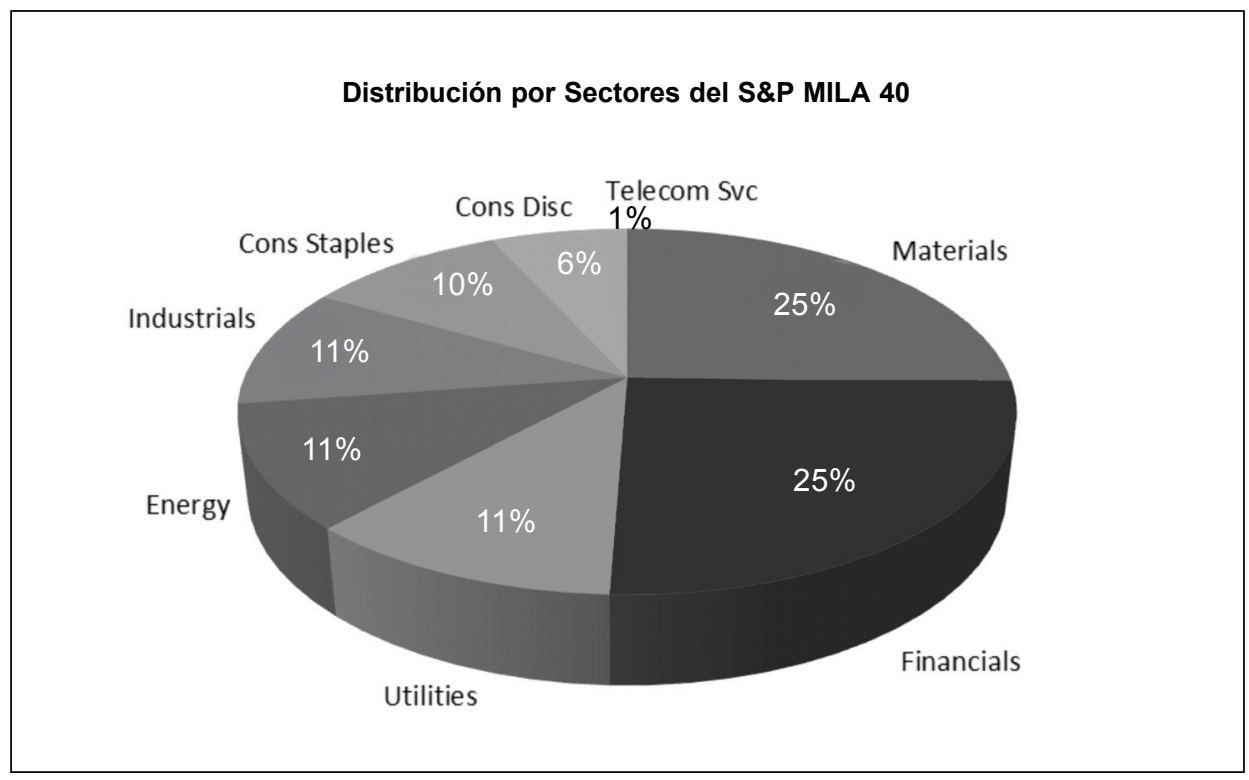

Gráfico 3. Distribución por Sectores del S\&P MILA 40. Fuente: MILA New, diciembre de 2011.

mercados con sistemas de liquidación o intercambio poco fiables generan desconfianza al inversionista (Martin, et al., 2006), los inversionistas están muy atentos a esta situación. La liquidación es el momento de la entrega de fondos y valores en simultaneo; es decir, cuando ocurre la entrega de fondos por parte del comprador y de los valores por parte del vendedor (Romero, et al., 2012), esta labor no la desempeñan las bolsas de valores sino los depósitos centrales, que son empresas privadas que cumplen la función de hacer la liquidación de las operaciones de compra y venta de productos financieros que se transan en el mercado bursátil y de la custodia o depósito de los títulos valores. En suma, una transacción puede realizarse en la plataforma de negociación, pero está sujeta a su perfeccionamiento. El comprador y el vendedor no se conocen y nunca tendrán ningún contacto; por tanto, los depósitos ayudan a que se realice el intercambio comercial.

De lo anteriormente dicho, los depósitos participantes de cada país en el MILA, son: DCV de Chile, Cavali de Perú y Deceval de Colombia. Estas tres entidades realizaron acuerdos de operatividad y de interconexión para armonizar las prácticas y el modelo de registro contable. Por ejemplo, el modelo de registro de DCV de Chile era distinto al que operaba en Colombia y en el Perú. Se realizaron los ajustes pertinentes para lograr la interconexión y que el flujo de la información fuese único, tanto para y como desde los tres mercados. Finalmente, con las tres empresas de depósitos y el apoyo de los reguladores, se logró establecer acuerdos que permitieron que fuera factible la liquidación de operaciones del registro de los valores comprados. 


\section{EFECTOS QUE HAN GENERADO DIFICULTADES AL MILA}

Ahora bien, al permitir la internacionalización de los mercados, la adquisición de acciones directamente de los mercados bursátiles foráneos presenta varios inconvenientes. Lo que primero observan los inversores cuando acuden a un mercado internacional es si el mercado goza de un buen nivel de desarrollo, como la existencia de barreras a la entrada de capital extranjero (Martin, et al., 2006). Para el caso del MILA, los gobiernos en sí no realizaron ningún cambio, sino que, simplemente, se remiten a indicar que se deben cumplir las normas vigentes en cada uno de los países. Para el caso de Colombia, el Banco de la Republica ejerce un control de cambios a través de unos reportes que exige a todas las compañías y entidades financieras cuando se hacen ingresos o salidas de dinero al país. Se busca determinar sobre todo la procedencia del dinero, en el sentido de cómo fue adquirido. Es importante resaltar que Colombia, de los tres países, es el que más control ejerce sobre movimientos de capitales extranjeros ${ }^{8}$. Este reporte se hace a partir de diez mil dólares y en Perú, en cambio, es a partir de cien mil dólares.

Al mismo tiempo, la igualación de los precios de los factores por la vía del comercio, es decir, la intensificación del comercio entre dos países, conduce a que la remuneración de los factores de la producción entre ambos tienda a igualarse. Sin embargo, este último concepto está sujeto a que los factores de la producción trabajo y capital tengan facilidad de movilidad. Esto es lo que ha empezado a ocurrir con la integración, que se realicen movimientos de capital a través de inversiones con la compra de acciones en los tres mercados.

Una segunda situación es la eficiencia operacional que se consigue con unos costos de transacción y comisiones de los brokers y spreads ${ }^{9}$ de los creadores de mercados bajos, (Martin, et al., 2006). Esta es un de las dificultades que tiene el mercado MILA. En primer lugar, los costos de las transacciones no fueron unificados en relación con los precios que cobran las bolsas de valores y los depósitos de valores. En segundo lugar, las comisiones de los brokers es imposible unificarlas porque ellos son empresas privadas independientes. Chile es el país donde son más costosas las comisiones de intermediación, seguido de Colombia y, por ultimo, Perú. Esto debería ser al contrario, por el desarrollo económico de Chile; pero en los dos años de operación del mercado no se han presentado cambios importantes en los precios.

Un tercer inconveniente es la eficiencia en la información. Esta se refiere al nivel en que los precios de los títulos valores reflejan determinadas informaciones, dificultad que puede considerarse como débil, semifuerte o fuerte (Martin, et al., 2006). Para el caso de un mercado débil,

8. Colombia ejerce un control fuerte en los movimientos de capitales extranjeros, fundamentalmente por razones derivadas del narcotráfico.

9. Es la diferencia entre el precio de compra y el precio de venta del precio de las acciones y está determinada por el número total de acciones en el mercado, el total de las operaciones diarias y la demanda de la acción en el mercado. 
solo emiten información histórica donde no se pueden predecir precios futuros. El mercado colombiano es donde más se presenta esta situación debido a que la información de las empresas es mínima, es histórica y de poca importancia. Primero, porque los canales de información no se actualizan oportunamente $y$, segundo, porque no se cuenta con un canal de información especial para este tipo de mercados. Para el caso de los mercados semifuertes, estos tienen aparte de los precios históricos información pública de las diferentes compañías. Mientras el mercado colombiano cuenta con la información de unas pocas empresas en esta sección, en Chile y Perú todas las empresas se encuentran en este rango. Por último, una información fuerte refleja la existencia de una información tanto pública como privada. De los tres mercados, el de Chile cuenta con unas cuantas empresas que presentan este tipo de información.

La principal dificultad que deben afrontar los inversionistas a la hora de tomar decisiones de inversión en el mercado MILA es el riesgo de tipo de cambio, todo ello como consecuencia de la incertidumbre en la evolución de la cotización que relaciona la moneda del inversor extranjero con la del mercado objetivo que se puede disminuir (Martin, et al., 2006). En el MILA, Chile utiliza pesos chilenos, Perú nuevo sol y Colombia pesos colombianos. Los inversionistas deberán cambiar su moneda local por la moneda del país donde desean invertir, pasando primero por la moneda patrón, el dólar americano. Por ejemplo, es necesario cambiar pesos colombianos a dólares y luego a pesos chilenos, cuando un inversionista colombiano desea comprar acciones en la BVS. Una vez termina la inversión, se hace la operación al contrario para llegar finalmente a pesos colombianos. Estas operaciones incrementan el riesgo y la incertidumbre en la compra de acciones en el mercado del MILA por la situación que a continuación exponemos.

El riesgo de transacción se deriva de los efectos que las alteraciones no previstas en el tipo de cambio pueden llegar a tener sobre los cobros y/o pagos aplazados en divisas (Bua, 2010). Los inversionistas de este mercado exponen su capital a operaciones de compra de acciones, en las que su valor está dado en monedas distintas a la de su país de origen y, una vez tomada la decisión de deshacer la inversión y retornar el capital al país de origen, muy seguramente la moneda y la divisa utilizadas no tendrán el mismo valor a la fecha inicial de la inversión.

Consecuentemente, las fluctuaciones de los tipos de cambio han logrado que aparezcan operaciones financieras que hacen que se minimicen estos riesgos, que se denominan coberturas. Las coberturas cambiarias son instrumentos que permiten comprar o vender divisas a futuro, pactando un precio y un monto determinado a un plazo acordado, minimizando de esta forma los riesgos asociados a la volatilidad del tipo de cambio. Por este motivo, no resulta extraño que los inversionistas estén destinando un gran esfuerzo -tanto en términos económicos como de recursos humanos -, a neutralizar los efectos adversos de la volatilidad cambiaria, fundamentalmente a través del uso intensivo de productos derivados. Según la encuesta realizada por el ISDA (International Swaps \& Derivatives Association), durante el año 2008 el 94\% de las empresas que forman parte del Fortune utilizaba productos derivados para la gestión del riesgo, destacándose los de divisas $(93,6 \%$ ) y los de tipo de interés $(6,4 \%)$ (Bua, et al., 2010). Para el caso de 
Colombia, según el diario La República, 2011, solo el 5\% de las empresas realizan operaciones de cobertura. En suma, las continuas fluctuaciones del tipo de cambio implica la volatilidad de los precios de una divisa generando, además, un riesgo significativo para cualquier inversionista, puesto que afecta adicionalmente el valor de la inversión.

Otro inconveniente es la homogenización de los datos contables, distorsionados por las diferencias en los principios y prácticas al uso de cada país. La armonización en el MILA está dada entre Chile y Perú al utilizar las normas internacionales de contabilidad (NIC). La dificultad está en Colombia, porque las empresas presentan los informes contables en normas contables nacionales.

La contabilidad es el sistema por el cual las empresas informan de su situación financiera a los entes reguladores, a los accionistas y al público en general. En especial, esto sucede cuando las empresas cotizan en una bolsa de valores, porque los inversionistas interesados en comprar acciones de una empresa desean conocer su situación financiera. Por consiguiente, los reportes contables son de vital importancia en el mercado de capitales. Ahora analizaremos la situación de los repostes contables que son exigidos por las tres bolsas de valores que hacen parte del MILA.

El cambio más significativo que se puede tener con las NIC es la utilización del valor razonable; este es un modelo de medición diferente al que se venía utilizando con las antiguas normas. Consiste en la valoración de los activos y pasivos de manera más cercana a su valor económico o valor de mercado; es decir, se busca permanentemente mantener el valor de los activos y pasivos en la medida en que transcurra el tiempo y se ajusten o se acerquen a su precio real del mercado actual.

En consecuencia, los inversionistas, para la toma de decisiones de inversión, utilizan los informes financieros que emiten las empresas; realizan, además, análisis fundamental ${ }^{10}$ con dichos informes, e incluso esto les permite hacer comparaciones, entender el comportamiento económico pasado de las empresas, conocer su endeudamiento, la rentabilidad, la capacidad operativa, entre otros. Los informes que se presentan actualmente en los tres países son similares, con la diferencia anotada anteriormente para el caso de Colombia, lo que realmente no es una dificultad para hacer los análisis y comparaciones respectivas. Sin embargo, es importante tener en cuenta el aspecto relacionado con el valor razonable. Por otro lado, al converger todos hacia las normas internacionales de contabilidad permite disponer de un set de normas contables globales que permitirán a los inversores de los diferentes países evaluar, bajo un denominador común, los diferentes mercados y oportunidades.

10. Se realiza análisis financiero de la empresa y la industria, y también un análisis de la economía en la que participa la empresa.

EL MILA. MERCADO DE INTEGRACIÓN ENTRE CHILE, PERÚ Y COLOMBIA 
Por último, los efectos fiscales son otra dificultad del MILA. La compra y venta de acciones en los mercados de valores conlleva a dos maneras de obtener ganancias por parte de inversionistas. La primera, cuando el precio de venta es mayor al de compra; la segunda, cuando el accionista recibe los dividendos ocasionados por la distribución de utilidades que se hacen generalmente cada año. Estas dos maneras de obtener ganancias originan que los inversores deban hacer unos pagos fiscales por enajenación de los productos financieros y por recibir parte de las utilidades de una empresa como accionistas. Adicionalmente, los contribuyentes en cada país están obligados a realizar declaración de renta anualmente por los ingresos que reciben, entre otros, por los ingresos obtenidos en operaciones de compra y venta de acciones. A continuación exponemos las normas que rigen cada país integrante del MILA para evitar la doble tributación y prevenir la evasión de impuestos.

Los países integrantes del MILA tenían convenios para evitar la doble tributación anterior a la conformación del mercado integrado y son los que rigen actualmente. Para empezar, en Perú y Colombia, la Decisión 578 de 2004, de la Comunidad Andina de Naciones, es aplicable a las personas domiciliadas en Colombia y Perú. En la relación Chile - Colombia, el convenio de doble tributación en Colombia se encuentra aprobado por la Ley 1261 de 2008 y en Chile mediante el Decreto Supremo No. 232 de 2009. Por último, en la relación Perú - Chile, el convenio para evitar la doble tributación en Chile aprobado mediante Decreto Supremo No. 297 de 2007 y en Perú mediante Resolución Legislativa No. 27905 de 2004 (Guía del MILA, 2012).

Los inversionistas colombianos, peruanos y chilenos cuando compran acciones del MILA deben de tener en cuenta que, cuando recibe dividendos de empresas peruanas, debe pagar el 4,1\%; cuando son empresas chilenas, el 35\% de impuesto; y cuando compran acciones de empresas colombianas, el 20\%, cuando es declarante y 35\% cuando no es declarante de impuestos. Para enajenación de acciones en Perú paga el 5\% y como máximo 18.000 soles para personas naturales; en Chile el $0 \%$ y en Colombia el 0\%. Para efectos de declaración de renta los colombianos tienen un impuesto del 33\% sobre los ingresos, en Perú deben pagar por ingresos de operaciones bursátiles el 6,25\% las personas naturales y el 30\% las personas jurídicas; y en Chile el 18,5\% para el 2012.

En síntesis, los impuestos por dividendos en Colombia y Chile son elevados, porque son del $33 \%$ y el 35\% respectivamente; en Perú, solo son el 4,1\%. Esto marca una gran diferencia cuando se desea invertir a largo plazo, porque para el inversionista que espera obtener ganancias por dividendos, Perú es la mejor plaza. Cuando se obtienen ganancias por incremento en el precio de la acción y, luego, cuando el inversionista vende las acciones, los impuestos en Colombia y en Chile son del $0 \%$ y en Perú son el $5 \%$ o el $30 \%$, si es persona jurídica. Realmente hay una pequeña diferencia con Perú que no es muy notable y que compensa con los dividendos en caso de ser una persona natural, pero si es persona jurídica sí marca una gran diferencia. Entonces, Perú deja de ser atractivo. En el caso del impuesto de renta, cada inversionista paga este 
impuesto en su país de origen. Cabe anotar que Colombia es el país más costoso, porque se paga el $33 \%$, seguido de Chile que paga el $18,5 \%$, y de Perú que paga el $6,5 \%$ si es persona natural y $30 \%$ si es persona jurídica.

\section{CONCLUSIONES}

La integración financiera ha estado siendo empujada por la expansión del comercio regional, así como de las inversiones y la liberalización de los sectores financieros domésticos. Para el caso específico del MILA, la integración de los mercados bursátiles representa para los inversionistas, empresarios e intermediarios un crecimiento importante del mercado, pero deben tener en cuenta algunas variables difíciles de controlar como el tipo de cambio. Los inversores, por eso, han de actuar teniendo en cuenta las distintas realidades económicas de cada país.

Para que esta integración se siga expandiendo en forma ordenada, la mayor contribución que pueden hacer los gobiernos es coordinar sus esfuerzos para mejorar la calidad de la información con respecto a los principales activos financieros que se transan en sus mercados. Esta mejora de la información se refiere al avance hacia la uniformización de los criterios contables, los tratamientos legales, los requerimientos de información y de su calidad.

La alianza es benéfica, a la vez, para los tres mercados, en la medida en que se cree una amplia cuenca de liquidez que permite dar acceso a más sociedades cotizadas y, también, porque diversifica la variedad de los instrumentos financieros cotizados hacia el futuro. La integración debe tratar por todos los medios de reducir los costos de acceso a los mercados para provocar, de esta forma, la disminución de los precios de las transacciones. Esto beneficia a los inversores, cuyos costos de gestión y de transacciones bajarán y, posteriormente, pueden realizar más fácilmente la diversificación internacional de sus carteras. La alianza, por último, beneficia también a los emisores permitiéndoles tasas de interés de financiación más bajas.

\section{REFERENCIAS}

- $\quad$ Adler \& Dumas. (1984). (Marcador De Posición 1). Exposure to Currency Risk: Definition and Measurement. Financial Management, 2, 41-50.

- Agacino, R. (1997). Anatomía de la Globalización y la integración económica, CEME, notas de discusión, 1-25.

- $\quad$ Arino, A \& de la Torre, C. (1998). Learning from failure: Towards an evolutionary model Of collaborative ventures. Organization science, 9, 306-325. 
- Balassa, B. (1980). Teoría de la integración económica. México: Fondo de Cultura Económica.

- Barney, J. B. (1991). Firm resources and sustained competitive advance. Harvard Business Review, 69 (6), 127-135.

- Beard, C., (1953). Una Interpretación Económica de la Constitución de los Estados Unidos. Buenos Aires: Arayu.

- Bua, V. (2010). El riesgo cambiario y su cobertura financiera. Revista Galegade economia, 6, 23-34.

- Brealey, R. Myers, S\& Allen, F. (2006). Principios de finanzas corporativas. (8 ed.). Mc Graw Hill, Madrid.

- Cline, W.R. \& Delgado, E. (1978). Economic Integration in Central America, Washington, The Brookings Institution.

- $\quad$ SIECA (1973). El Desarrollo Integrado de Centroamérica en la presente década, Buenos Aires, INTAL. 11 volúmenes.

- Conesa, E. R. (1981). Aplicabilidad de la tarifa optima en Argentina. Revista Integración Latinoamericana, 62, 6-22.

- Corbo, V. (1997). Integración financiera en América latina. Documentos de trabajo de la oficina de economista jefe. Banco Interamericano de Desarrollo, 1-26.

- $\quad$ Corbo, V. \& Hernández, C (1993).What Should Banks Do in the Financial Industry of the 1990s. En S. Faruqui (ed.), Financial sector reforms in Asian and Latin American countries (pp. 2-34). The World Bank. Washington, USA..

- $\quad$ Corden, W. M. (1972). Economies of Scale and Customs Union Theory. Journal of Political Economy, 80, 465-475.

- Chi, T. (1994). Trading in strategic resources: Necessary condicions, transation Cost problems, and choice of exchange structure. Strategic Management Journal, 15, 271-290.

- Chile, B. C. (2012). Informe de politica monetaria. Recuperado de http://www.bcentral.cl/

- Dobson, W. y Hufbauer, G. C. (2001). World Capital Markets: Challenges to the G-10. Peterson Institute. Nevada, USA. 
- Dyer, S \& Singh, C (1998). The relation view: cooperative stragic and sources of interorgacinal competitive advantage. Academy of management review, 23, 660-679.

- $\quad$ Enderlein, H. (2011). The economic impact of the Deutsche Borse-Nyse Euronex Merger on the European financial markets. Hertie school of governance,Vol 1, pp 66.

- $\quad$ Flood, E. and Lessard, D. (1986). On the Measurement of Operating Exposure to Exchanges Rates: A Conceptual Approach. Financial Management, 15, (1), 25-37.

- $\quad$ FMI (1997, may). Globalization, Opportunities and Challenges. World Economic Outlook, 4-12.

- $\quad$ García, C. (1999). Desarrollando estrategias competitivas. Icade, 48, 99-117.

- Gun, C. y Resnick, B. (2006). Estrategias de crecimiento, Administración financiera internacional, (3era Ed.), Bogotá, Colombia.: Mc Graw Hill. (pp.155- 176).

- $\quad$ Haspeslagh, B \& Jemison, D. (1991). Managing acquisitions. New Yorkl, 45,16-20.

- Hernnart, J. (1988). A transaction cost theory of equity joint venture. Strategic management journal. 9, 361-374.

- Hekman, C. (1983). Measuring Foreign Exchange Exposure: A Practical Theory and Its Application. Financial Analysts Journal, 23, 59-65.

- Hennar, M \& Reddy, F. (1997). The choice between mergers acquisitions and joint ventures: The case of Japanese investors in the united states. Strategic Management Journal, 18, $1-12$.

- Hill, D \& Jones, A (2009). Administración estratégica (8ª Ed.). Mc Graw Hill, Barcelona, España.

- $\quad$ Kern, S. (2008). EU-US financial market integration. Deutche Bank Research, 16, 1-27.

- Madhok, H \& Tallman, G. (1998). Resources, transactions and rents: Managing value Through interfirm collaborative relationships. Organization Science, 9, 326-339.

- Machlup, Fritz. (1977). A History of Thought on Economic Integration. New York: Columbia University Press. 
- Mateus, J \& Brasset, C, (2002). La integración financiera, Revista Economía y Desarrollo, 1, 62- 66 .

- $\quad$ Noel, L. M. \& Veiga, L. (2008). El tipo de cambio. Revista abc de Economía, 6, 80- 85.

- $\quad$ Peteraf, M. (1993). The cornerstones of competitive advantage: A resource-based view. Strategic Management Journal, 14, 179-191.

- $\quad$ Rialp, J \& Salas, M. (2002). La colaboración empresarial desde la teoría de los derechos de propiedad. Investigaciones Económicas, XXVI (1), 113-144.

- Ricardo, D. (1959). Principios de Economía Política y Tributación. México: Fondo de Cultura Económica.

- $\quad$ Ring, K \& Van de Ven, J (1994). Developmental processes of cooperative interorganizational relationships. Academy of Management Review, 19, 90-118.

- $\quad$ Robson, P.(2003). Integration among Centrally Planned Economies, 12, 128-144.

- $\quad$ Romero, F. (2012). El Mila: Palanca de financiamiento para el desarrollo (pp. 11-154). Depósito legal en la Biblioteca Nacional del Perú.

- Samuelson, P. A. (1986). International factor-price equalization once again. Economic Journal, 59, 23-35. 